\title{
On Maps Preserving Unitarily Invariant Norms of the Spectral Geometric Mean
}

\author{
Hongjie Chen1, Lei Li' ${ }^{2}$, Zheng Shi' ${ }^{1}$ Liguang Wang1* \\ ${ }^{1}$ School of Mathematical Sciences, Qufu Normal University, Qufu, China \\ ${ }^{2}$ School of Mathematical Sciences and LPMC, Nankai University, Tianjin, China \\ Email: chenhongjiehei@163.com, leilee@nankai.edu.cn, sz17865513508@163.com, ^wangliguang0510@163.com
}

How to cite this paper: Chen, H.J., Li, L., Shi, Z. and Wang, L.G. (2021) On Maps Preserving Unitarily Invariant Norms of the Spectral Geometric Mean. Journal of Applied Mathematics and Physics, 9, 577-583.

https://doi.org/10.4236/jamp.2021.94041

Received: March 6, 2021

Accepted: April 12, 2021

Published: April 15, 2021

Copyright $\odot 2021$ by author(s) and Scientific Research Publishing Inc. This work is licensed under the Creative Commons Attribution International License (CC BY 4.0).

http://creativecommons.org/licenses/by/4.0/

\begin{abstract}
We consider maps on positive definite cones of von Neumann algebras preserving unitarily invariant norms of the spectral geometric means. The main results concern Jordan ${ }^{*}$-isomorphisms between $C$-algebras, and show that they are characterized by the preservation of unitarily invariant norms of those operations.
\end{abstract}

\section{Keywords}

Spectral Geometric Mean, Positive Cone, Jordan *-Isomorphisms, Unitarily Invariant Norm

\section{Introduction}

In order to study the geometry on the cones $\mathbb{P}_{n}$ of the positive definite $n \times n$ matrices, people consider different means of positive definite matrices. Szokol, Tsai and Zhang [1] investigated the structure of geodesic affine maps in $\mathbb{P}_{n}$ and considered the following three types of geodesics:

- The weighted arithmetic mean

$$
\gamma_{A, B}^{h}(t)=(1-t) A+t B, \quad t \in[0,1], A, B \in \mathbb{P}_{n} .
$$

- The weighted geometric mean

$$
\gamma_{A, B}^{g}(t)=A \#_{t} B=A^{\frac{1}{2}}\left(A^{-\frac{1}{2}} B A^{-\frac{1}{2}}\right)^{t} B^{\frac{1}{2}}, \quad \forall t \in[0,1], A, B \in \mathbb{P}_{n} .
$$

- The weighted log-Euclidean mean

$$
\gamma_{A, B}^{l}(t)=\exp ((1-t) \log A+t \log B), \quad \forall t \in[0,1], A, B \in \mathbb{P}_{n} .
$$

The authors in [1] considered the maps preserving $\gamma_{A, B}^{a}(t) \quad(a \in\{h, g, l\})$ 
under the $p$-norm for some $1 \leq p<\infty$. They showed that those maps are the restriction of algebra ${ }^{*}$-automorphisms and ${ }^{*}$-antiautomorphisms on $\mathbb{M}_{n}$. Furthermore, Gaál and Nagy ([2], Theorem 1) obtained the same results as in [1] concerning the bijective transforms of $\mathbb{P}_{n}$ which preserve any unitary invariant norm of some quasi-arithmetric means of elements (it includes the weighted arithmetic mean and the weighted log-Euclidean mean) for all $t \in[0,1]$. For the log-Euclidean mean, the Gaál and Nagy ([2], Theorem 2) obtained a general results concerning the $p$-norm in a $C$-algebra $/$ equipped with a faithful tracial state, where $p \in[1, \infty)$. All the results showed that any correspondence preserver is the restriction of a Jordan ${ }^{*}$-isomorphism of $/$ multiplied by a central positive invertible element [3] [4]. Molnár and Szolol ([5], Theorem 2) considered those bijective maps between the positive semidefinite cones of standard operator algebras which preserve some given symmetric norm of a rather general Kubo-Ando operator mean of the elements.

Suppose that $\mathscr{C}$ is a $C$-algebra, then we denote by $\mathscr{C}^{++}$the set of all positive invertible elements in ./, which is called the positive definite cone of d . The spectral geometric mean is the operation on $/^{++}$defined by

$$
A \sigma_{s} B=\left(A^{-1} \# B\right)^{1 / 2} A\left(A^{-1} \# B\right)^{1 / 2}, \quad \forall A, B \in \mathscr{C}^{++},
$$

where

$$
A \# B=\sqrt{A}\left(\frac{1}{\sqrt{A}} B \frac{1}{\sqrt{A}}\right)^{\frac{1}{2}} \sqrt{A} .
$$

It requires some algebraic manipulations to verify that $\sigma_{s}$ (which is not a Kubo-Ando type mean) is also symmetric (see [6]). We refer to [7] for more details about spectral geometric mean. Concerning a map preserving the spectral geometric mean, we have only for the full operator algebra over a Hilbert space.

Theorem 1.1. (see ([7], Theorem 3)) Let $\mathscr{H}$ be a complex Hilbert space and let $\phi: \mathcal{B}(\mathscr{C})^{++} \rightarrow \mathcal{B}(\mathscr{K})^{++}$be a continuous bijective map such that

$$
\phi\left(A \sigma_{s} B\right)=\phi(A) \sigma_{s} \phi(B), \quad \forall A, B \in \mathcal{B}(\mathscr{C})^{++} .
$$

If $\phi(I)=I$ and $\phi$ has a continuous bijective extension to $\mathcal{B}(\mathscr{H})^{++}$, then there is a unitary or antiunitary operator $U$ on $\mathscr{C}$ such that $\phi(A)=U A U^{*}$ for all $A \in \mathcal{B}(\mathscr{H})^{++}$.

For the $C$-algebra norm, Proposition 7 in [7] showed that:

Theorem 1.2. Let $\mathscr{C}, \mathscr{B}$ be $C$-algebras. Let $\phi: \mathscr{C}^{++} \rightarrow \mathscr{B}^{++}$be a surjective map. Then

$$
\left\|\phi(A) \sigma_{s} \phi(B)\right\|=\left\|A \sigma_{s} B\right\|, \quad \forall A, B \in \mathscr{C}^{++}
$$

if and only if there is a Jordan ${ }^{*}$-isomorphism $J: \mathcal{C} \rightarrow \mathscr{B}$ which extends $\phi$.

In this present note, we consider maps on positive definite cones of $C$-algebras or von Neumann algebras preserving unitarily invariant norms of the spectral geometric means.

Let $/$ be a $C$-algebra and $N$ a norm on . We say that $N$ is unitarily 
invariant if $N(U A V)=N(A)$ for all unitaries $U, V \in \mathscr{C}$ and $A \in \mathscr{C}$. We say a norm $N$ on $\mathscr{C}$ has the property $P$ if $A, B \in \mathscr{C}^{++}, A \leq B$ and $N(A)=N(B)$ implies $A=B$. Let $/ /$ be a von Neumann algebra with a faithful tracial state $\tau$ and let $1 \leq p<\infty$. It can be verified that the function

$$
A \mapsto\left(\tau\left(|A|^{p}\right)\right)^{\frac{1}{p}}
$$

defines a unitary invariant norm on $/ /$ (see ([8], Section 3)). Moreover, Molnár ([9], Lemma 4) proved that the above $p$-norm with $1 \leq p<\infty$ has the property $(\mathrm{P})$.

\section{Main Results}

Let $\mathscr{A}$ be a $C$-algebra with a unitarily invariant mean $N$. Let $A, B \in \mathscr{C}^{++}$. It follows from [7] that $A \sigma_{s} B$ is unitarily congruent to $(\sqrt{A} B \sqrt{A})^{\frac{1}{2}}$ and therefore $N\left(A \sigma_{s} B\right)=N\left((\sqrt{A} B \sqrt{A})^{\frac{1}{2}}\right)$.

Lemma 2.1. Let $\mathscr{C}$ be a $C$-algebra with a unitarily invariant mean $N$ having property $P$. Let $A, B \in \mathscr{C}^{++}$. Then $A \leq B$ iff for any $X \in \mathscr{I}^{++}$, $N\left(X \sigma_{s} A\right) \leq N\left(X \sigma_{s} B\right)$.

Proof. $(\Rightarrow)$ Let $A, B \in \mathscr{I}^{++}$and suppose $A \leq B$. For any $X \in \mathscr{C}^{++}$, we have

$$
\sqrt{X} A \sqrt{X} \leq \sqrt{X} B \sqrt{X}
$$

and therefore

$$
(\sqrt{X} A \sqrt{X})^{\frac{1}{2}} \leq(\sqrt{X} B \sqrt{X})^{\frac{1}{2}}
$$

Then by the proof of Proposition 3 in [10], we have

$$
N\left((\sqrt{X} A \sqrt{X})^{\frac{1}{2}}\right) \leq N\left((\sqrt{X} B \sqrt{X})^{\frac{1}{2}}\right)
$$

and this implies $N\left(X \sigma_{s} A\right) \leq N\left(X \sigma_{s} B\right)$.

$(\Leftarrow)$ Suppose for any $X \in \mathscr{C}^{++}, N\left(X \sigma_{s} A\right) \leq N\left(X \sigma_{s} B\right)$. Our aim is to show $A \leq B$. Let $P$ be the spectral projection of $B-A$ corresponding to $(-\infty, 0]$. Then $P B P \leq P A P$ and $\sqrt{P B P} \leq \sqrt{P A P}$. Hence

$$
N(\sqrt{P B P}) \leq N(\sqrt{P A P}) \text {. }
$$

But we also have

$$
N(\sqrt{P A P}) \leq N(\sqrt{P B P})
$$

(since for any $X \in \mathscr{C}^{+}, \varepsilon>0$,

$$
N\left((X+\varepsilon I) \sigma_{s} A\right) \leq N\left((X+\varepsilon I) \sigma_{s} B\right)
$$

and let $\varepsilon \rightarrow 0$, we get $\left.N\left(X \sigma_{s} A\right) \leq N\left(X \sigma_{s} B\right)\right)$ and therefore 


$$
N(\sqrt{P B P})=N(\sqrt{P A P}) .
$$

Since $N$ has property $P$, we have $\sqrt{P B P}=\sqrt{P A P}$ and therefore $P B P=P A P$, i.e., $P(B-A) P=0$. It follows that $A \leq B$.

We also need the following result in [11].

Lemma 2.2. Let $/$ be a von Neumann algebra and $N$ is a unitarily invariant norm on $/$ with property $(P)$. Let $\alpha \in(0,1)$ be fixed. For $A, B \in \mathscr{I}^{++}$, we have $A \leq B$ if and only if $N\left(A \#_{\alpha} X\right) \leq N\left(B \#_{\alpha} X\right)$ for all $X \in \mathscr{V}^{++}$.

Theorem 2.3. Suppose $/$ and are von Neumann algebras with unitarily invariant norms $N$ and $M$ respectively and both having property (P). Let $\phi: \mathscr{C}^{++} \rightarrow \mathscr{B}^{++}$be a bijective map. If

$$
M\left(\phi(A) \sigma_{s} \phi(B)\right)=N\left(A \sigma_{s} B\right), \forall A, B \in \mathscr{C}^{++},
$$

then there is a Jordan ${ }^{\star}$-isomorphism $J: \mathscr{A} \rightarrow \mathscr{B}$ and an element $C \in \mathscr{B}{ }^{++}$ such that $\phi(A)=C J(A) C$ for all $A \in \mathscr{C}^{++}$.

Proof. We first show that $\phi$ is positive homogeneous. Indeed, for any $A, B \in \mathscr{C}^{++}, t>0$, we have

$$
(t A) \sigma_{s} B=t^{\frac{1}{2}}\left(A \sigma_{s} B\right)
$$

and therefore

$$
\begin{aligned}
M\left((t \phi(A)) \sigma_{s} \phi(B)\right) & =M\left(t^{\frac{1}{2}} \phi(A) \sigma_{s} \phi(B)\right)=t^{\frac{1}{2}} N\left(A \sigma_{s} B\right) \\
& =N\left(\left(t^{\frac{1}{2}} A\right) \sigma_{s} B\right)=M\left(\phi(t A) \sigma_{s} \phi(B)\right) .
\end{aligned}
$$

This implies that $\phi(t A)=t \phi(A)$ by Lemma 2.1 and $\phi$ is positive homogeneous. Since for any $A, B \in \mathscr{C}^{++}$, we have

$$
\begin{aligned}
A \leq B & \Leftrightarrow \forall X \in{\mathscr{\nearrow ^ { + + }}}^{+}, N\left(X \sigma_{s} A\right) \leq N\left(X \sigma_{s} B\right) \\
& \Leftrightarrow \forall X \in{\mathscr{\nearrow ^ { + + }}}^{+}, M\left(\phi(X) \sigma_{s} \phi(A)\right) \leq M\left(\phi(X) \sigma_{s} \phi(B)\right) \\
& \Leftrightarrow \forall Y \in \mathscr{P}^{++}, M\left(Y \sigma_{s} \phi(A)\right) \leq M\left(Y \sigma_{s} \phi(B)\right) \\
& \Leftrightarrow \phi(A) \leq \phi(B),
\end{aligned}
$$

$\phi$ is an order isomorphism. It follows from [10] that there exist an element $C \in \mathscr{B}^{++}$and a Jordan ${ }^{\star}$-isomorphism $J: \mathscr{A} \rightarrow \mathscr{B}$ such that

$$
\phi(A)=C J(A) C, \quad \forall A \in \mathscr{I}^{++} .
$$

Note that we have

$$
M\left((\sqrt{C J(A) C} C J(B) C \sqrt{C J(A) C})^{\frac{1}{2}}\right)=N\left((\sqrt{A} B \sqrt{A})^{\frac{1}{2}}\right)
$$

and $M(C J(A) C)=N(A)$ for all $A, B \in \mathscr{C}^{++}$. Also we have $M\left(C^{2}\right)=N(I)$.

Remark 2.3. Suppose $\mathscr{C}, \mathscr{B}$ are von Neumann algebras with unitarily invarinat norms $N$ and $M$ having property $(\mathrm{P})$. When there is a Jordan 
*-isomorphism $J: \mathscr{C} \rightarrow \mathscr{B}$ and a central element $C \in \mathscr{B}{ }^{++}$such that $\phi(A)=C J(A) C$ and $M(C J(A) C)=N(A)$ for all $A \in \mathscr{C}^{++}$, it is easy to check that

$$
M\left(\phi(A) \sigma_{s} \phi(B)\right)=N\left(A \sigma_{s} B\right), \quad \forall A, B \in \mathscr{\nearrow}^{++}
$$

Corollary 2.4. Suppose $\mathscr{C}$ and $\mathscr{B}$ are von Neumann algebras. Assume that $\mathscr{C}$ is a factor with a unique tracial state $\operatorname{Tr}$ and $\tau$ is a tracial state of $\mathscr{P}$. Let $1 \leq p<\infty$ and $\|\cdot\|_{p}$ the $p$-norm corresponding to $\operatorname{Tr}$ and $\tau$. Suppose $\phi: \mathscr{C}^{++} \rightarrow \mathscr{P}^{++}$is a bijective map such that

$$
\left\|\phi(A) \sigma_{s} \phi(B)\right\|_{p}=\left\|A \sigma_{s} B\right\|_{p}, \quad \forall A, B \in \mathscr{d}^{++} .
$$

Then $\mathscr{B}$ is also a factor and there is a bijective linear map $\Phi: \mathscr{C} \rightarrow \mathscr{B}$ which is an algebra ${ }^{*}$-isomorphism or ${ }^{*}$-antiisomorphism such that $\Phi(A)=\phi(A)$ for all $A \in \mathscr{C}^{++}$.

Proof. The proof is similar to that of Corollary 5 in [10] and we omit it.

Theorem 2.5. Suppose $\mathscr{C}$ and $\mathscr{P}$ are von Neumann algebras with unitarily invariant norms $N$ and $M$ both having property $(\mathrm{P})$. Let $\phi: \mathscr{C}^{++} \rightarrow \mathscr{R}^{++}$be a bijective map. If

$$
M\left(\phi(A) \sigma_{s} \phi(B)\right)=N(A \# B)
$$

holds for all $A, B \in \mathscr{C}^{++}$, then there exist a Jordan ${ }^{*}$-isomorphism $J: \mathscr{C} \rightarrow \mathscr{P}$ and an element $C \in \mathscr{P}^{++}$such that $\phi(A)=C J(A) C$ for all $A \in \mathscr{C}^{++}$.

Proof. We first show that $\phi$ is positive homogeneous. Indeed, for any $A, B \in \mathscr{C}^{++}, t>0$, we have

$$
(t A) \sigma_{s} B=t^{\frac{1}{2}}\left(A \sigma_{s} B\right),(t A) \# B=t^{\frac{1}{2}}(A \# B) .
$$

Therefore

$$
\begin{aligned}
M\left((t \phi(A)) \sigma_{s} \phi(B)\right) & =M\left(t^{\frac{1}{2}}(\phi(A)) \sigma_{s} \phi(B)\right)=t^{\frac{1}{2}} M\left((\phi(A)) \sigma_{s} \phi(B)\right) \\
& =t^{\frac{1}{2}} N(A \# B)=N((t A) \# B)=M\left(\phi(t A) \sigma_{s} \phi(B)\right)
\end{aligned}
$$

and this implies that $\phi(t A)=t \phi(A)$. Hence $\phi$ is positive homogeneous.

For any $A, B \in \mathscr{C}^{++}$, we have

$$
\begin{aligned}
A \leq B & \Leftrightarrow \forall X \in A^{++}, N(X \# A) \leq N(X \# B) \\
& \Leftrightarrow M\left(\phi(X) \sigma_{s} \phi(A)\right) \leq M\left(\phi(X) \sigma_{s} \phi(B)\right) \\
& \Leftrightarrow \forall Y \in B^{++}, M\left(Y \sigma_{s} \phi(A)\right) \leq M\left(Y \sigma_{s} \phi(B)\right) \\
& \Leftrightarrow \phi(A) \leq \phi(B)
\end{aligned}
$$

(note the first equivalence follows from Lemma 2.2) and therefore $\phi$ is an order isomorphism. It follows from [10] that there exist an element $C \in \mathscr{P}^{++}$ and a Jordan ${ }^{*}$-isomorphism $J: \mathscr{C} \rightarrow \mathscr{B}$ such that $\phi(A)=C J(A) C$ for all $A \in \mathscr{\gamma}^{++}$. 


\section{Conclusion}

Mean is an important concept in mathematics. There are many interesting results studying preserver transformations relating operator means. In this paper, we show that maps on positive definite cones of von Neumann algebras preserving unitarily invariant norms of the spectral geometric means can be characterized by Jordan ${ }^{\star}$-isomorphisms.

\section{Acknowledgements}

The authors would like to thank the anonymous referee for constructive criticisms and valuable comments.

\section{Founding}

Partially supported by NFS of China $(11871303,11971463,11671133)$ and NSF of Shandong Province (ZR2019MA039 and ZR2020MA008).

\section{Conflicts of Interest}

The authors declares no conflicts of interest regarding the publication of this paper.

\section{References}

[1] Szokol, P., Tsai, M.C. and Zhang, J. (2015) Preserving Problems of Geodesic-Affine Maps and Related Topics on Positive Definite Matrices. Linear Algebra and Its Applications, 483, 293-308. https://doi.org/10.1016/j.laa.2015.06.009

[2] Gaál, M. and Nagy, G. (2018) Preserver Problems Related to Quasi-Arithmetic Means of Invertible Positive Operators. Integral Equations Operator Theory, 90, Article No. 7. https://doi.org/10.1007/s00020-018-2426-x

[3] Molnár, L. (2019) Quantum Rényi Relative Entropies on Density Spaces of $\mathrm{C}^{*}$-Algebras: Their Symmetries and Their Essential Difference. Journal of Functional Analysis, 277, 3098-3130. https://doi.org/10.1016/j.jfa.2019.06.009

[4] Molnár, L. (2020) Spectral Characterization of Jordan-Segal Isomorphisms of Quantum Observables. Journal of Operator Theory, 83, 179-195.

[5] Molnár, L. and Szokol, P. (2015) Transformations Preserving Norms of Means of Positive Operators and Nonnegative Functions. Integral Equations Operator Theory, 83, 271-290. https://doi.org/10.1007/s00020-015-2241-6

[6] Fiedler, M. and Pták, V. (1997) A New Positive Definite Geometric Mean of Two Positive Definite Matrices. Linear Algebra and Its Applications, 251, 1-20. https://doi.org/10.1016/0024-3795(95)00540-4

[7] Li, L., Molnár, L. and Wang, L. (2021) On Preservers Related to the Spectral Geometric Mean. Linear Algebra and Its Applications, 610, 647-672. https://doi.org/10.1016/j.laa.2020.10.014

[8] Nelson, E. (1974) Notes on Non-Commutative Integration. Journal of Functional Analysis, 15, 103-116. https://doi.org/10.1016/0022-1236(74)90014-7

[9] Molnár, L. (2018) Maps between the Positive Definite Cones of Operator Algebras Preserving a Norm of a Geodesic Correspondence. Acta Scientiarum Mathematicarum (Szeged), 84, 451-463. https://doi.org/10.14232/actasm-018-514-x 
[10] Hatori, O. and Molnár, L. (2014) Isometries of the Unitary Groups and Thompson Isometries of the Spaces of Invertible Positive Elements in $\mathrm{C}^{*}$-Algebras. Journal of Mathematical Analysis and Applications, 409, 158-167. https://doi.org/10.1016/j.jmaa.2013.06.065

[11] Chabbabi, F., Mbekhta, M. and Molnár, L. (2020) Characterizations of Jordan *-Isomorphisms of $\mathrm{C}^{*}$-Algebras by Weighted Geometric Mean Related Operations and Quantities. Linear Algebra and Its Applications, 588, 364-390.

https://doi.org/10.1016/j.laa.2019.11.024 